\title{
LA EXPRESIÓN DEL ASPECTO VERBAL DURATIVO. MODALIDADES DE TRANSFERENCIA LINGÜÍSTICA EN DOS ÁREAS DEL ESPAÑOL DE AMÉRICA
}

La finalidad de estas páginas es la descripción y análisis de dos fenómenos, homólogos, de transferencia lingüística verificados, respectivamente, en la zona andina suramericana, en la que están (o han estado) en situación de contacto lingüístico el español y las diversas variantes territoriales de la lengua quechua ${ }^{1}$, y en el área guaranítica del mismo continente ${ }^{2}$ en que las lenguas que se encuentran en dicha situación han sido (y son aún) el español y el guaraní.

El rasgo lingüístico concreto de que me ocuparé es identificable con el referido a la marcación morfosintáctica explícita del aspecto verbal durativo en las lenguas quechua y guaraní y a la incidencia que esta circunstancia ha determinado en las modalidades de español que se encuentran, desde hace más de cuatro siglos, en estrecho e íntimo contacto con aquéllas. La consideración pormenorizada de las peculiaridades de este pro-

${ }^{1}$ Sobre este tema véanse, sobre todo, A. Torero, "Los dialectos quechuas", Anales Científicos de la Universidad Agraria, 2 (1964), 446-478 y "La familia lingüística quechua", en América Latina en sus lenguas indígenas, ed. B. Pottier, Monte Ávila, Caracas, 1983, pp. 61-92. Últimamente R. CERRÓN-PALOMINo, Lingüistica quechua, Centro de Estudios Rurales Andinos "Bartolomé de las Casas", Cuzco, 1987.

${ }^{2}$ Sobre la extensión de la misma véase G. DE Granda, Sociedad, historia y lengua en el Paraguay, Instituto Caro y Cuervo, Bogotá, 1988 y, más específicamente, la n. 80 (pp. 367-368) del estudio "El contacto lingüístico como factor de retención gramatical. Aportes a su estudio sobre datos del área guaranítica suramericana”, en el volumen del autor Español de América, español de África y hablas criollas hispánicas, Gredos, Madrid, 1994, pp. 337-368. En el presente trabajo me ocuparé, sin embargo, con exclusividad de datos procedentes del guaraní y del español paraguayos dejando así, de lado, los que se refieren a ambas lenguas en las zonas argentinas y bolivianas que son incluibles también dentro del área guaranítica. 
ceso puede, en mi opinión, no sólo aportar datos valiosos para la fijación de la fisonomía areal, histórica y contemporánea, que debe ser atribuida a las mencionadas modalidades diatópicas de español americano ${ }^{3}$, sino también colaborar, mediante el examen de algunos de los factores condicionadores del hecho estudiado, a la determinación de relevantes aspectos teóricos relacionados con los fenómenos de transferencia lingüística.

La marcación gramatical del aspecto verbal durativo está presente en numerosas lenguas amerindias ${ }^{4}$ entre las que se incluyen tanto el quechua como el guaraní, pero, si hay entre estas últimas una clara coincidencia en cuanto a la expresión en ellas de la mencionada categoría por medio de elementos morfológicos específicos, no la hay, por el contrario, ni en lo que se refiere a las peculiaridades concretas que revisten los morfemas en cuestión, ni tampoco en lo que toca a los parámetros que en cada una de las lenguas mencionadas determinan la variabilidad formal de los mismos.

En quechua el aspecto verbal durativo se expresa mediante cuatro sufijos verbales derivativos diferentes identificables, en cuanto a sus formas originarias, respectivamente con $y k a$ :, $-\check{c} k a:,-k u \mathrm{y}-\check{s} k i$.

El primero de ellos (que también se empleó, al parecer, en las variedades diacrónicas cuzqueña y costera de quechua descritas por González Holguín y fray Domingo de Santo Tomás) es utilizado en las modalidades diatópicas del centro-norte del Perú incluyendo las de San Martín, Pastaza y Ucayali, si bien en algunos puntos de la provincia de Cajatambo (Lima) y de Jauja (Junín) el morfema base $-y k a$ : ha pasado a $-y y a$, mientras que en las provincias de Junín, Yauli, Tarma, buena parte de los distritos de Jauja y Huancayo (Junín) y, en parte, en el quechua de Huallaga se ha simplificado en $y a$ :.

El morfema verbal durativo -cka: se usa en las variedades meridionales del quechua, al sur de Huancavelica, aunque, en todos los casos, sin alargamiento vocálico. Por otra parte (salvo en algunas hablas puneñas y en el área al norte de La Paz) en toda la zona la marca originaria ha perdido la $[\mathrm{k}]$ derivando, alternativamente, $\mathrm{a}-s^{y} a,-\check{s} a$ o $-s a$.

${ }^{3}$ Cf. sobre este particular G. DE GRANDA, "El influjo de las lenguas indoamericanas sobre el español. Un modelo interpretativo sociohistórico de variantes areales de contacto lingüístico", Revista Andina (en prensa).

${ }^{4}$ Así, por ejemplo, en wichi (mataco). Cf. M. T. Viñas UrQuiza, Lengua mataca, Universidad, Buenos Aires, 1974, t. 1, p. 75. 
La marca durativa verbal $-k u$ es utilizada actualmente en el quechua ecuatoriano, colombiano y napeño, una vez sustituido su valor originario (mediopasivo) en el que fue reemplazada por $-r i$, anteriormente de significación inceptiva.

Finalmente, el morfema -ški se emplea en el quechua de Ferreñafe y en las provincias de Ancash y Huánuco que bordean el río Marañón si bien en parte de estas zonas el valor durativo del mismo ha sido alterado apreciablemente ${ }^{5}$.

En cuanto a la expresión gramatical del aspecto verbal durativo en guaraní debemos diferenciar en ella dos modalidades, la primera corresponde a los registros ${ }^{6}$ superiores de esta lengua y la segunda a los inferiores, caracterizables por su índole de discurso vernáculo ${ }^{7}$. En aquélla se utiliza para indicar dicho aspecto un morfema, pospuesto al verbo, constituido (salvo en tercera y sexta personas) por el elemento -ina precedido de las correspondientes marcas personales, las cuales, por lo tanto, se reiteran, ya que también han sido utilizadas, previamente, como prefijos de las formas verbales que reciben el morfema durativo en cuestión ${ }^{8}$. En cuanto a las personas verbales tercera y sexta, la marca verbal durativa es, en ambos casos, -hina, aunque en el último se emplea también, en competencia con -hina, la marca -hikuái ${ }^{9}$.

${ }^{5}$ Cf. el volumen de R. Cerrón-Palomino ( supra, n. 1) pp. 145-148, 193 y 213-215, para una visión general del tema. También, entre otros trabajos atinentes a este punto, G. J. PARKer, Derivación verbal en el quechua de Ancash, CILA, Lima, 1973 y V. MannheIm, "Southern Peruvian Quechua”, en South American Indian languages: Retrospect and prospect, eds. H. Klein, \& L. Stark, University of Texas, Austin, 1985, pp. 481-515 y L. STARK, "The Quechua language in Bolivia”, en el mismo volumen, pp. 516-545.

${ }_{6}^{6}$ Sobre este concepto teórico (correspondiente al término inglés styles) véanse W. Labov, Modelos sociolingüísticos, Cátedra, Madrid, 1983, pp. 115-125; J. URE, y J. ElLIS, "El registro en la lingüística descriptiva y en la sociolingüística actual”, en La sociolingüistica actual, ed. O. Uribe Villegas, UNAM, México, 1974, pp. 115-164 y A. BELL, "Language style as audience design”, LangS, 13 (1984), 145-204.

7 Véanse las precisiones que expone L. MiLroy en relación con esta designación caracterizadora en Observing and analyzing natural language. A critical account of sociolinguistic method, Basil Blackwell, Oxford, 1987, pp. 57-58.

8 Así en agueru'aina 'yo estoy trayendo', reguerureina 'tú estás trayendo', jagueruñaina 'nosotros [inclusivo] estamos trayendo', peguerupeína 'ustedes están trayendo'. Sobre el valor aspectual durativo de -ina véase S. Liuzzi, y P. KirTchuк, "Tiempo y aspecto en Guaranî”, Amerindia, 1989, núm. 14, 9-42.

9 Así en ogueruhína 'él/ellos está/están trayendo', ogueruhikuái 'ellos están trayendo’. Véase, para una descripción muy completa de este paradig- 
En la modalidad vernácula o casual $^{10}$ del actual guaraní paraguayo se utiliza para marcar el aspecto durativo un solo morfema, invariable en todas las personas verbales, -hina, extendiendo así a las demás la marca que en el registro alto del guaraní corresponde sólo a las personas tercera y sexta ${ }^{11}$.

De la exposición que hasta aquí hemos realizado respecto a las modalidades de expresión del aspecto verbal durativo en quechua y en guaraní se deduce que la marcación, común a ambas lenguas, de dicha categoría gramatical por medio de elementos morfológicos específicos de superficie ofrece, sin embargo, notorias diferencias en dos aspectos importantes.

En primer lugar (y como ya lo afirmábamos, tangencialmente, más arriba) en quechua la variabilidad de los morfemas aspectuales durativos depende, claramente, de condicionamientos de índole territorial, diatópica, mientras que en guaraní paraguayo, por el contrario, a la unidad geográfica que caracteriza, en toda la extensión del país, al mencionado paradigma verbal corresponde, inversamente, una evidente dualidad de realizaciones, determinada por factores de índole diastrática del mismo.

Pero, además, también existe entre ambas lenguas, por lo que toca al rasgo que aquí examinamos, una notoria diferenciación en cuanto a la fisonomía formal que presentan, en cada una de ellas, los morfemas durativos.

En efecto, en quechua estos morfemas son siempre, por su posición en el lexema verbal, de índole interna y ligada ya que están precedidos por la raíz y seguidos, cuando menos, en el mismo, por las marcas correspondientes de persona ${ }^{12}$. Contra-

ma verbal y de sus posibilidades alternativas (por ejemplo, el uso de los morfemas verbales, también alternativos, aikóvo, reikóvo, iokóvo, jakóvo/roikóvo, peikóvo, oikóvo), A. GUASCH, El idioma guarani. Gramática y antología de prosa y verso, Asunción, 1976, pp. 123-124.

10 Sobre esta última determinación del concepto de registro véase F. MoRENo Fernández, Metodología sociolingüistica, Gredos, Madrid, 1990, pp. 66-67.

11 Cf. N. Krivoshein de CANese, Gramática de la lengua guarani, Asunción, 1983, pp. 99-100. La gramática guaraní del P. ANTONIO GuASch (cf. supra, n. 9) no recoge esta modalidad de expresión del aspecto verbal durativo en el guaraní actual, sin duda por el enfoque extremadamente purista que la caracteriza.

12 Véanse, como ejemplos, miku-čka-n, del quechua de Ayacucho, o mik$h u-s a-n$, de los dialectos sureños ('él está comiendo'), en que - $n$ es, en los dos casos, marca personal mientras que miku / mikhu es la raíz verbal y -čka- / sa- el morfema aspectual durativo. Del mismo modo, en las formas 
riamente, en guaraní (en sus dos modalidades diastráticas) son por su posición final, sufijada, de carácter externo e incluso, en el registro vernáculo o causal, el elemento hína (único marcador verbal durativo existente en él) posee características claras de morfema independiente o no ligado, lo cual, como más adelante veremos en detalle, es de gran importancia como factor determinador del proceso de transferencia en situaciones de contacto lingüístico ${ }^{13}$.

Muy otra es, obviamente, la situación que se da, respecto al rasgo que nos ocupa, en la lengua española. En ella, del mismo modo que en el resto de las lenguas románicas ${ }^{14}$, salvo alguna curiosa excepción ${ }^{15}$, la categoría de aspecto ${ }^{16}$ no tiene expresión explícita propia en el paradigma verbal sino que se marca en él de modo simultáneo a los valores de tiempo y modo17, dando lu-

$\overline{m i k u-k u-n i}$ (del quechua colombiano) o miku-yka-ni (del quechua de Cajamarca) 'yo estoy comiendo', los morfemas durativos $-k u$ - e $-y k a$ - son precedidos por la raíz verbal y seguidos por -ni, marca de primera persona.

${ }_{13} \mathrm{Cf}$., sobre el rol diferencial de los elementos morfológicos internos/externos y ligados/no ligados en situaciones de contacto de lenguas, N. Dorian, Language death: The life cycle of a Scottish Gaelic dialect, University of Pennsylvania, Philadelphia, 1981, y C. CLAIRIS, "Le processus de disparition des langues", LingP, 27 (1991), 3-13.

14 Cf. E. Coseriu, Das romanische Verbalsystem, Tübingen, 1976. También J. ŠABRŠUla, "Contribution aux problèmes de méthode de la recherche dans le domaine de l'aspect verbal (langues romanes)", en Actes du Xe Congrès International de Linguisitique et Philologie Romanes, C. Klincksieck, Paris, 1965, t. 1, pp. 157-173.

${ }_{15}$ Me refiero, en concreto, a la existencia en istrorrumano de un amplio subsistema verbal de aspectos morfológicamente marcados. Cf. A. KovAčEC, Descrierea istroromânei actuale, Bucarest, 1971. Obviamente se trata, en este caso, de un proceso de transferencia desde las lenguas eslavas (que lo rodean totalmente) al aislado y muy minoritario dialecto istrorrumano.

16 Sobre este concepto véase en especial B. Comrie, Aspect, Cambridge University, London, 1978, aunque sigue siendo útil J. J. MaCLennan, El problema del aspecto verbal, Gredos, Madrid, 1962. Cf. también J. DAvid, et R. MARTin (eds.), La notion d'aspect, Metz, 1980; P. Tedeschi, and A. Zaenen (eds.), Syntax and semantics; tense and aspect, Academic, New York, 1981; C. BAcHE, "Aspect and aktionsart: Towards a semantic distinction", $J L, 18$ (1982), 5772 y D. DowTHIE, “Tense and aspect in discourse”, LiP, 9 (1986).

17 Cf., en cuanto a diferentes enfoques de esta temática, K. Togeby, Mode, aspect et temps en espagnol, Ejnar Munksgaard, Kobenhaun, 1953; M. SÁNCHEZ RuiPÉREz, "Observaciones sobre el aspecto verbal en español”, en Strenae. Homenaje a M. García Blanco, Universidad, Salamanca, 1962, pp. 427-435; J. Cerny, "Sobre la asimetría de las categorías del tiempo y del aspecto en el verbo español", PhP, 12 (1969), 83-93; C. RALlides, The tense-aspect system of the Spanish verb, Mouton, Paris, 1971; N. Cartagena, "Acerca de las categorías de 
gar, así, a un sistema muy diferente, en este particular, al utilizado, por ejemplo, en griego, árabe, o en las lenguas eslavas, en todas las cuales la categoría aspectual ocupa una posición de gran relevancia en la articulación interna del sintagma verbal.

De este modo, la marcación específica del aspecto en español se ve limitada al empleo de los que denomina F. Marcos Marín ${ }^{18}$ modificadores secundarios y terciarios, es decir, de formas verbales perifrásticas ${ }^{19}$. Las que, de entre ellas, se constituyen con gerundio son portadoras de aspecto durativo o, como lo denomina C. Hernández Alonso, cursivo-imperfectivo ${ }^{20}$.

Así pues, podemos considerar que expresiones del tipo de estar, venir, ir, seguir, etc. + gerundio constituyen, en castellano, el único sintagma gramatical que marca explícitamente, con exclusividad, el contenido significativo de duración verbal ${ }^{21}$ aun-

tiempo y aspecto en el sistema verbal del español", Revista de la Sociedad Española de Lingüística, 8 (1978), 373-408; J. SLAwOMIrSky, "La posición del aspecto en el sistema verbal español”, Revista de la Sociedad Española de Lingüistica, 13 (1983), 91-119, y G. Rojo, "Temporalidad y aspecto en el verbo español", LEA, 10 (1988), 195-216. También deben verse, entre otros trabajos atinentes al tema J. RocA Pons, "El aspecto verbal en español", Linguistica Antverpiensia, 2 (1968), 385-399; S. MARINER BigORRA, "Triple noción básica en la categoría modal castellana”, RFE, 54 (1971), 209-252; L. TовóN de Castro y J. Rodríguez Rendón, "Algunas consideraciones sobre el aspecto verbal en español”, BICC, 29 (1974), 34-47; J. M. Guitart, "Aspects of Spanish aspect", en Contemporary studies in Romance linguistics, ed. M. Suñer, Georgetown University, Washington, 1978, pp. 132-168; J. P. RonA, "Tiempo y aspecto: análisis binario de la conjugación española”, ALM, 11 (1973), 211223; C. Silva-Corvalán, "A speech event analysis of tense and aspect in Spanish", en Proceedings of the XIIth Linguistic Symposium on Romance Languages, J. Benjamins, Amsterdam, 1984, pp. 229-251 y, de la misma autora, "Tense and aspect in oral Spanish narrative: Context and meaning”, Lan, 59 (1983), 760780. Y, para terminar, deben tenerse en cuenta muy especialmente E. ALARCOS LLORACH, Estudios de gramática funcional del español, Gredos, Madrid, 1973 y C. Hernández Alonso, Gramática funcional del español, Gredos, Madrid, 1986, pp. 360-379.

18 Curso de gramática española, Cincel, Madrid, 1980, pp. 270-280.

19 Cf. acerca del tema, J. Roca Pons, Estudios sobre perífrasis verbales del español, C.S.I.C., Madrid, 1958; M. LaunaY, "Acerca de los auxiliares y frases verbales", LEA, 2 (1980), 39-79; L. Gómez Torrego, Perífrasis verbales, Arco, Madrid, 1988; F. Fernández de Castro, Las perífrasis verbales en español, Oviedo, 1990 y M. Veyrat Rigat, Perifrasis, aspecto y auxiliación, Valencia, 1993. Un excelente modelo teórico de ámbito románico facilita W. DieTRICH, El aspecto verbal perifrástico en las lenguas románicas, Gredos, Madrid, 1983.

${ }^{20}$ Op. cit., pp. 390-391.

${ }^{21}$ Cf. S. Fernández Ramírez, "Algo sobre la forma estar + gerundio", en Homenaje a Dámaso Alonso, Gredos, Madrid, 1960, t. 2, pp. 509-516; J. W. 
que, en cada caso, con matizaciones expresivas que aún no han sido bien estudiadas ${ }^{22}$.

Una vez que hemos expuesto, con la brevedad obligada, las características más destacadas que, en relación con el rasgo gramatical que aquí nos ocupa, están presentes en las gramáticas de las lenguas en contacto en las áreas andina y guaranítica de Suramérica, es decir en quechua, guaraní y español ${ }^{23}$, es tiempo de precisar ya qué modalidades de transferencia se han producido en cuanto a la expresión del aspecto verbal durativo en las zonas consideradas, respectivamente, desde el quechua y el guaraní al español local andino y paraguayo.

En cuanto al primero de los casos mencionados, y coincidiendo en ello con otros especialistas entre los que se encuentran, por ejemplo, R. Nardi ${ }^{24}$, Ch. Kany ${ }^{25}$ y J. A. Hasler ${ }^{26}$, estimo

ZDNECK, "Another look at the progressive", H, 55 (1972), 498-499 y L. KING, $\&$ M. SuñER, "The meaning of progressive in Spanish and Portuguese", The Bilingual Review, 7 (1980), 222-239. Se refieren a este tipo de construcciones perifrásticas en determinadas áreas diatópicas americanas E. LunA TrAILL, "Perífrasis de gerundio en el habla culta de México", en Estudios sobre el español hablado en las principales ciudades de América, ed. J. M. Lope Blanch, UNAM, México, 1977, pp. 201-208 y G. B. STOne, "Las perífrasis verbales de gerundio en el habla culta de La Habana”, ALM, 22 (1984), pp. 195-207.

${ }^{22}$ Representa, en este sentido, un buen planteamiento teórico A. MARTÍNEZ DE LóPEz, "Las frases verbales con gerundio a la luz de un sistema tempo-modal-aspectual”, ALHis, 6 (1990), 325-333. Véase también K. ZaGONA, "Las perífrasis de gerundio y la reestructuración”, RALM, 2 (1986), 231-244.

${ }^{23}$ Deberíamos, quizá, incluir también en nuestro análisis comparativo a la lengua aimara que, en amplias zonas andinas de Bolivia, Perú e incluso de Chile, está igualmente en contacto con el español local. Bástenos aquí senalar (dejando para otra ocasión la debida consideración del tema) que en aimara, de modo muy similar a lo que ocurre en quechua, se marca explícitamente por medios morfológicos el aspecto verbal durativo empleando para ello el elemento originario -ska- (y sus alomorfos diatópicos). Cf. J. CARVAJAL, Apuntes para una morfología aymara, Instituto Nacional de Estudios Lingüísticos, La Paz, 1983; M. HaRdman, Compendio de estructura fonológica y gramatical del aymara, Instituto Nacional de Estudios Lingüísticos, La Paz, 1983; E. H. Martín, Bosquejo de estructura de la lengua aymara, Universidad, Buenos Aires, 1969; M. Grondin, Método de aymara, Editores Rodríguez-Muriel , Oruro, 1973; J. Herrero, D. Cotari, y J. Mejía, Lecciones de aymara, Instituto de Idiomas, Cochabamba, 1969 y, en cuanto a variabilidad de tipo diatópico, L. T. BRIGGS, "Dialectal variation in Aymara", South American Indian languages..., pp. 595-616.

24 "Lenguas en contacto: el substrato quechua en el noroeste argentino",

Fil, 17/18 (1976-77), 131-150 (esp. p. 148).

25 Sintaxis hispanoamericana, Gredos, Madrid, 1976, p. 284.

26 "Morphemes of Southern quechua and their influence on Spanish", 
que el coeficiente, extraordinariamente alto, de uso de formas perifrásticas de gerundio (como reemplazo de las formas verbales simples equivalentes) que se encuentra en español andino puede ser relacionado causalmente con la presión sobre el mismo del modelo gramatical quechua, en cuya estructura verbal, como sabemos, existía, con marca morfológica propia, el aspecto durativo.

Fundamento mi apreciación en dos líneas argumentativas, geográfica la una y la otra sociolingüística, además, obviamente, de referirme, como punto de partida común a ambas, a la homología funcional de índole lingüística a que acabo de aludir.

Parece, en efecto, evidente que, desde un enfoque territorial, el rasgo lingüístico que aquí analizamos, es decir la intensa sustitución por perífrasis verbales de gerundio, con valor durativo, de las formas verbales simples equivalentes ${ }^{27}$, coincide de modo perfecto en su distribución geográfica con las zonas $s e-$ rranas de las repúblicas andinas suramericanas desde Bolivia al área meridional de Colombia pasando por Perú ${ }^{28}$ y Ecuador ${ }^{29}$ e incluyéndose también en este ámbito diatópico el noroeste argentino ${ }^{30} \mathrm{o}$, en otras palabras, con los territorios en que se da aún o se ha dado históricamente el contacto lingüístico español-quechua (o, en su caso, español-aimara).

Y, por otra parte, según se deduce de los trabajos que se han ocupado de este tema desde parámetros diastráticos ${ }^{31}$, es notoriamente más intenso el fenómeno lingüístico en cuestión en

en Approaches to language, eds. W. McCormack, \& S. A. Wurm, The Hague-Paris, 1978, pp. 235-242 (esp. pp. 241-242) y El quichua meridional y su influjo en el español criollo, Grupo de Autores, Cali, 1984.

${ }_{27} \mathrm{Me}$ refiero a expresiones del tipo está viniendo (= viene), me vengo olvidando (= me olvido), estás pudiendo (= puedes), me he de estar vendiendo (= venderé), ahi viene llegando (= ahí llega), han de estar llegando (= llegarán), etcétera.

${ }^{28}$ Cf. R. CARAvedo, "Espacio geográfico y modalidades lingüísticas en el español del Perú", en Historia y presente del español de América, Valladolid, 1992, pp. 719-741.

${ }^{29}$ Cf. H. Toscano Matéus, El español en el Ecuador, C.S.I.C., Madrid, 1953.

${ }^{30}$ Véanse el trabajo de R. NARDi (cit. supra, n. 24) y el de G. DE GRANDA, "Quechua y español en el noroeste argentino. Una precisión y dos interrogantes", Lexis, 17 (1993), 259-274.

31 Véanse, sobre todo, J. G. MendozA, "El castellano hablado en Bolivia", en Historia y presente..., pp. 437-499 (esp. pp. 462-463, 468, 472 y 487) y J. C. Godenzzi, "Lengua y variación sociolectal. El castellano en Puno", Pesquisas en lingüistica andina, ed. L. E. López, Lima-Puno, 1988, pp. 201-236. 
las variedades vernáculas o en los registros inferiores de las modalidades dialectales consideradas lo que, dada la mayor presencia y vigor de la lengua quechua (o aimara) en estos segmentos sociológicos de las comunidades estudiadas, parece apuntar directamente al establecimiento de una clara conexión causal entre esta última circunstancia y el rasgo lingüístico que aquí analizamos.

Ahora bien, si (en mi opinión, al menos) la valoración objetiva tanto de las características funcionales del fenómeno lingüístico en cuestión en las lenguas española y quechua como de las circunstancias geográficas y sociológicas de que acabamos de hacer mención parece sustentar firmemente la hipótesis de que en la génesis del rasgo gramatical que nos ocupa ha participado un proceso de transferencia propiciado por el contacto lingüístico quechua-castellano, considero igualmente como posible que este factor causal no sea exclusivo sino que haya actuado sólo como condicionante parcial en una situación de causación múltiplę2.

Así lo hace pensar el hecho, ya apuntado por Ch. Kany ${ }^{33}$, de que las perífrasis verbales de contenido aspectual - y entre ellas las perífrasis con gerundio, de valor durativo- hayan sido más abundantes en el español medieval e incluso clásico que en el moderno ${ }^{34}$, como ha ocurrido también en otras lenguas románicas $^{35}$, lo que, si se toma debidamente en cuenta la abundancia de arcaísmos lingüísticos que caracteriza al español andino ${ }^{36}$, conduce a postular para el rasgo que analizamos un condicionamiento genético parcial, no exclusivo, relacionable con un muy posible proceso de retención gramatical compatible, desde luego, con el factor causal, derivado del contacto quechuaespañol, propuesto anteriormente en estas mismas páginas.

32 Sobre este concepto teórico véanse Y. MALkiel, "Multiple versus simple causation in linguistic change", en To Honor R. Jakobson, Mouton, The Hague, 1967, t. 2, pp. 1228-1246 y "On hierarchizing the components of multiple causation”, StLang, 1, (1987), 81-108.

33 Loc. cit. (supra, n. 25).

34 Cf. A. Yllera, Sintaxis histórica del verbo español. Las perífrasis medievales, Universidad, Zaragoza, 1980 y J. LARochetTE, "Les aspects verbaux en espagnol ancien", RLR, 68 (1939), pp. 345 ss.

35 Véase M. WANDruszka, Nuestros idiomas: comparables e incomparables, Gredos, Madrid, 1976, pp. 524-525.

36 Cf., por ejemplo, P. YABAR, "Regionalismos del Departamento de Ancash (Perú)”, en Estudios sobre el léxico del español de América, ed. M. Perl, Ver- 
Una vez que hemos diseñado, hasta aquí, las principales notas caracterizadoras del fenómeno de transferencia lingüística que ha tenido lugar en el área andina en relación con el tema de estudio, debemos ocuparnos, a continuación, de la fisonomía del proceso, homólogo en cuanto a su sentido y direccionalidad, que ha tenido lugar, en el área guaranítica, como resultado del prolongado e intenso contacto lingüístico que se ha dado en ella entre el guaraní y el español.

De modo apreciablemente desemejante a lo que, en relación con el rasgo concreto que analizamos, aconteció en la zona andina según hemos visto, el proceso de transferencia referido a la marcación del aspecto verbal durativo que se ha desarrollado en el área guaranítica es claro, inequívoco y desprovisto de las facetas inferenciales y, en cierto sentido al menos, hipotéticas que están presentes en aquél.

El español paraguayo (en sus registros más próximos al polo vernáculo) ha adoptado e incorporado, por lo tanto, a su estructura verbal el marcador morfológico de aspecto durativo existente en el guaraní local, hina, con el mismo valor que posee en la lengua prestataria, como yo mismo lo anoté en alguna otra ocasión (si bien muy de pasada) ${ }^{37}$ y como también lo han expuesto otros estudiosos ${ }^{38}$.

Ahora bien, téngase en cuenta que el mencionado morfema procede, como se deduce de sus características de elemento formalmente invariable y no ligado ${ }^{39}$, de la modalidad diastrática

lag Enzyklopädie, Leipzig, 1982, pp. 161-175 y el volumen de H. ToscANo (cit. supra, n. 29, passim).

37 Así en mis trabajos "Préstamos morfológicos del guaraní en el español del Paraguay. I", recogido ahora en volumen en G. DE GRANDA, Sociedad, historia y lengua en el Paraguay, Instituto Caro y Cuervo, Bogotá, 1988, pp. 167181 (esp. p. 176) y "El español del Paraguay. Distribución, uso y estructuras", en Historia y presente..., pp. 675-695 (esp. p. 692).

38 Cf. B. Usher de Herreros, "Castellano paraguayo. Notas para una gramática contrastiva castellano-guaraní”, Suplemento Antropológico. Universidad Católica, 11 (1976), 29-123 (esp. pp. 67-68).

${ }^{39}$ En mis cuadernos de notas de campo correspondientes a mis largos periodos de residencia en el país encuentro, entre otros, los siguientes ejemplos del uso de hína en español paraguayo: "aqui trabajo hína todo el verano"; "Ilueve hina el fin de semana"; "el muchacho estudia hina en este colegio"; "¿que hacés aquí?- "y lloro hina"; "leo hína desde que vos te fuiste", "cuando Juan le dejó [a ella] María lloró mucho hína”. La última frase transcrita no deja, por cierto, duda sobre el carácter independiente, no ligado, del morfema hína en español (y guaraní) paraguayo. Compárese, por otra parte, la absoluta corres- 
causal o vernácula del guaraní y no de los registros superiores de dicha lengua en los que el morfema de aspecto verbal durativo es, contrariamente, variable en cuanto a la expresión de la persona sujeto y, además, dependiente o ligado ${ }^{40}$.

Habiendo presentado en las páginas anteriores las notas más importantes que caracterizan los fenómenos de transferencia respecto a la marcación del aspecto verbal durativo desarrollados, respectivamente, en las áreas andina y guaranítica suramericanas debemos ahora analizar, comparativamente, las mismas con vistas a dos finalidades concretas: la fijación de sus respectivas fisonomías tipológicas desde un enfoque descriptivo, de índole teórica, que las relacione con los modelos generales clasificatorios de las diferentes modalidades de transferencia en situaciones de contacto lingüístico y la determinación de los factores causales que han propiciado, en cada caso considerado, las peculiaridades caracterizadoras de su específica modalidad de proceso de transferencia lingüística.

Refiriéndonos al primero de los enfoques que acabamos de mencionar, constatamos que los dos fenómenos de transferencia analizados en estas páginas manifiestan numerosas discordancias tipológicas entre sí al mismo tiempo que algunas (escasas) coincidencias.

Entre estas últimas deben ser resaltadas las referidas a la índole del proceso de contacto lingüístico que ha tenido lugar en ambas áreas el cual, partiendo de la conceptualización elaborada por S. G. Thomason y T. Kaufman ${ }^{41}$, debe ser definido como de sustitución lingüistica (language shift) y no de préstamo lingüistico (linguistic borrowing) ${ }^{42}, \mathrm{y}$, también, a la direccionalidad (transferencia) y al nivel de esta última (morfosintáctico), coincidentes en las dos zonas consideradas.

En cuanto a las discordancias que se dan entre los dos fenómenos de transferencia lingüística analizados, son identificables las siguientes, que expondré muy sucintamente:

pondencia existente entre los valores aspectuales de hina en las dos expresiones siguientes: omba'apo hína kokuépe - él trabaja hína en la capuera ('él está trabajando en la capuera').

${ }^{40}$ Cf. supra, notas 8 y 9.

${ }^{41}$ Cf. su obra Language contact, creolization and genetic linguistics, University of California, Berkeley, 1988. También G. R. GuY, "The sociolinguistic types of language change", Diachronica, 7 (1990), 47-67.

42 Cf. supra, n. 3. 
— En el área andina ha tenido lugar, por lo que toca al rasgo que aquí hemos analizado, un caso de convergencia del español respecto al quechua mientras que en la guaranítica se ha producido un hecho de interferencia del guaraní sobre el español local ${ }^{43}$.

- En la zona andina se ha dado un fenómeno de ampliación de uso en cuanto al empleo de las perífrasis durativas con gerundio del español. En la guaranítica un fenómeno de adopción por el español del morfema verbal durativo del guaraní (hína).

- En territorio andino el quechua ha ejercido muy probablemente sobre el español, en cuanto al rasgo que nos ocupa, una acción concomitante en situación de causación múltiple. En el guaranítico, y en relación con el mismo rasgo, el guaraní ha actuado sobre el español de modo exclusivo.

- Por lo que se refiere al rasgo gramatical que analizamos, el fenómeno de transferencia verificado en el área andina implica solamente una modificación en la distribución de un elemento gramatical del español. El fenómeno, homólogo, producido en la zona guaranítica ha conducido, contrariamente, a la modificación del inventario morfológico del español local ${ }^{44}$.

- Como corolario del punto anterior, el resultado del proceso de transferencia que nos ocupa ha determinado un coeficiente de relevancia (saliency) considerablemente mayor en el área guaranítica que en la andina ${ }^{45}$.

43 Sobre los conceptos teóricos de transferencia, convergencia e interferencia lingüísticas véase ahora G. DE GRANDA, "Interferencia y convergencia lingüísticas e isogramatismo amplio en el español paraguayo", Español de América, español de África y hablas criollas hispánicas. Cambios, contactos y contextos, Gredos, Madrid, 1994, pp. 314-336.

${ }^{44}$ Véase, sobre esta temática teórica, M. SALA, El problema de las lenguas en contacto, UNAM, México, 1988.

45 Acerca de este concepto véanse P. Trudgill, Dialects in contact, Blackwell, Oxford, 1976, pp. 11 ss. y A. NAro, "The social and structural dimensions of a syntactic change”, Lan, 57 (1981), 63-98. Una aplicación concreta del mismo a la diacronía del español americano se realiza en G. DE Granda, "Formación y evolución del español de América. Época colonial", en Español de America, español de África y..., pp. 49-103 (esp. pp. 83-84). 
- Puede ser considerado, a su vez, como consecuencia de la notable relevancia que reviste el resultado gramatical del fenómeno de transferencia aquí analizado en el área guaranítica y, por el contrario, de la más reducida que el mismo adquiere en la andina el diferente grado de integración de ambos en el español local de los territorios implicados, mayor sin duda (aunque sería necesario estudiar a fondo el tema, de ser posible con procedimientos cuantificadores) en la zona andina que en la paraguaya ${ }^{46}$.

Las notas diferenciales hasta aquí apuntadas respecto al rasgo lingüístico que estudiamos coinciden en líneas generales (en cuanto a la peculiaridad que presentan las áreas andina y guaranítica respecto a la incidencia en el español de fenómenos de transferencia lingüística originados, respectivamente, por el contacto con las lenguas quechua y guaraní) no sólo con las conclusiones de un reciente trabajo de nuestra autoría en el que se analizaba otro proceso, homólogo en buena parte al aquí estudiado $^{47}$, sino también con los resultados, más o menos explícitos, de investigaciones anteriores sobre la misma temática general ${ }^{48}$.

En este sentido, parece quizá indicada la caracterización de las tendencias mayoritarias (no exclusivas, desde luego) que han actuado, por lo que toca a procesos de transferencia lingüística en las áreas andina y paraguaya de español, como referidas, respectivamente, a la producción de calcos funcionales ${ }^{49}$, conducentes en buena parte de los $\operatorname{casos}^{50}$ a fenómenos de conver-

${ }^{46}$ Sobre la noción de integración como diferente de la interferencia en situaciones de contacto lingüístico véase W. F. MAскEY, Bilinguisme et contact des langues, Klincksieck, Paris, 1976. Sobre el concepto de grado de integración cf. Ll. PAYRATó, La interferència lingüistica. (Comentaris i exemples catalá-castellá), Barcelona, 1985.

47 "Dos procesos de transferencia gramatical de lenguas amerindias (Quechua-Aru y Guaraní) al español andino y al español paraguayo. Los elementos validadores", RFE (en prensa).

${ }^{48}$ Véanse, por ejemplo, en lo que toca a la zona andina, los estudios de R. Cerrón-Palomino, "Calcos sintácticos en el castellano andino", San Marcos, 14 (1976), 93-101; "Aspectos sociolingüísticos y pedagógicos de la motosidad en el Perú”, en Temas de lingüistica amerindia, Lima, 1990, pp. 153-180 y, en cuanto al área paraguaya, Sociedad, historia y ..., passim, pero, en esp. pp. 167-203 y 249-306, y "El español del Paraguay. Distribución, uso y estructuras”, en Español de América, español de África y..., pp. 288-313.

${ }^{49}$ Sobre este concepto teórico véase R. KonTzi, "Calcos semánticos en textos aljamiados", en Actas del Coloquio Internacional sobre Literatura Aljamiada y Morisca, Gredos, Madrid, 1978, pp. 315-336.

${ }^{50}$ Para ejemplos de ello, cf. supra, notas 30 y 47 y, también, parcial- 
gencia quechua-español, y a la adopción de elementos gramaticales de la lengua guaraní de contacto.

En lo que toca a los factores determinadores que, en cada una de las zonas mencionadas han dado lugar a la génesis de la situación apuntada, creo adecuado reiterar aquí la posición teórica que ya esbocé en ocasión anterior 51 .

Sin perjuicio de las precisiones que un análisis individual pormenorizado pueda facilitar respecto a cada caso específico de transferencia de lenguas amerindias desarrollado en las áreas andina y paraguaya de español, estimo adecuado centrar la individualización de los mismos en los parámetros dimensionales siguientes: densidad distribucional considerablemente mayor en guaraní que en quechua de elementos morfológicos independientes o no ligados ${ }^{52}$; situación similar en lo que respecta a morfemas de caracterización posicional externa (normalmente sufijada, como corresponde a la índole tipológica de las lenguas quechua y guaraní) respecto a los de carácter interno ${ }^{53}$ y, como consecuencia de las dos circunstancias mencionadas, existencia de un índice de transparencia funcional ${ }^{54}$ notoriamente más elevado en guaraní que en quechua, lo que es considerado, de modo general, por los estudiosos más notorios de esta temática ${ }^{55}$, como factor altamente relevante en la producción de fenómenos de transferencia en situaciones de contacto lingüístico.

GERMÁn de GRANDA Universidad de Valladolid

mente, R. Cerrón-Palomino, "La forja del castellano andino o el penoso camino de la ladinización”, en Historia y presente..., pp. 201-234, véanse los estudios citados supra, n. 48.

51 Cit. supra, n. 47.

52 En relación con la importancia de este punto en cuanto a la ampliación o reducción de fenómenos de transferencia interlingüística, véase $\mathrm{V}$. Weinreich, Languages in contact, Mouton, The Hague, 1970, pp. 29-46.

${ }^{53} \mathrm{Cf}$., sobre esta circunstancia y su incidencia en los procesos de contacto lingüístico, supra, notas 13 y 47.

54 Cf., para la exposición de este concepto teórico, P. Kiparsky, "Historical linguistics", en A survey of linguistic science, ed. W. O. Dingwall, University of Maryland, College Park, 1971, pp. 476-562 y "Abstractness, opacity and global rules", en The application and ordering of grammatical rules, ed. A. Koutsoudas, Mouton, The Hague, 1976, pp. 160-186.

55 Cf., sobre todo, D. Slobin, "Language change in childhood and history", Language learning and thought, ed. J. Macnamara, Academic, San Francisco, CA, 1977, pp. 185-214 y, como enfoque general, H. H. Hock, Principles of historical linguistics, Mouton de Gruyter, Berlin, 1986. 